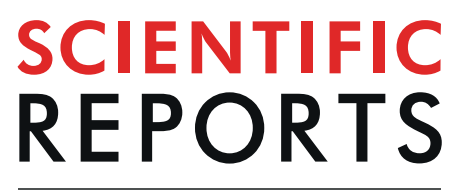

natureresearch

\title{
Adapting SureSelect enrichment protocol to the lon Torrent S5 platform in molecular diagnostics of craniosynostosis
}

\author{
Ewelina Bukowska-Olech $\mathbb{1}^{1,2}{ }^{2}$, Delfina Popiel ${ }^{3}$, Grzegorz Koczyk $\mathbb{D}^{3,4}$, Anna Sowińska-Seidler $\mathbb{D}^{1}$, \\ Magdalena Socha $\circledast^{1}$, Bartosz Wojciechowicz ${ }^{5}$, Adam Dawidziuk ${ }^{3}$, Dawid Larysz ${ }^{6}$ \& \\ Aleksander Jamsheer $\left(\mathbb{1}^{1,3^{*}}\right.$
}

Obtaining reliable and high fidelity next-generation sequencing (NGS) data requires to choose a suitable sequencing platform and a library preparation approach, which both have their inherent assayspecific limitations. Here, we present the results of successful adaptation of SureSelect hybridisationbased target enrichment protocol for the sequencing on the lon Torrent S5 platform, which is designed to work preferably with amplicon-based panels. In our study, we applied a custom NGS panel to screen a cohort of 16 unrelated patients affected by premature fusion of the cranial sutures, i.e. craniosynostosis (CS). CS occurs either as an isolated malformation or in a syndromic form, representing a genetically heterogeneous and clinically variable group of disorders. The approach presented here allowed us to achieve high quality NGS data and confirmed molecular diagnosis in $19 \%$ of cases, reaching the diagnostic yield similar to some of the published research reports. In conclusion, we demonstrated that an alternative enrichment strategy for library preparations can be successfully applied prior to sequencing on the lon Torrent $S 5$ platform. Also, we proved that the custom NGS panel designed by us represents a useful and effective tool in the molecular diagnostics of patients with CS.

\section{Next-Generation Sequencing (NGS) in Medical Genetics}

Routine NGS diagnostics requires high-quality sequencing data, short turnaround time and reasonable cost of the investigations. Therefore, out of the three major NGS-based diagnostic strategies, i.e. whole exome sequencing (WES), whole genome sequencing (WGS), and targeted gene panel sequencing, the final approach is ubiquitous and broadly applied in the clinical settings ${ }^{1-5}$. Successful implementation of targeted NGS in medical diagnostics results from several advantages. First, it generates disease-restricted data with fewer variants of uncertain significance, simplifying the analysis. Next, it provides very high coverage and read depth of selected regions, and finally, it limits the need for expensive laboratory equipment and data storage $e^{6,7}$. In order to generate reliable, high fidelity NGS data one has to choose a suitable sequencing platform and a library preparation protocol ${ }^{6,8}$. Different NGS platforms are known to have their specific limitations, such as underrepresentation of sequences with high guanine-cytosine (GC) content in case of Illumina or homopolymer length estimation bias in Ion Torrent semiconductor-based sequencing systems ${ }^{9-14}$. In addition to dissimilarities of NGS platforms and their specific inbuilt artefacts, also the sample preparation protocols differ in many aspects, including enrichment strategy. Currently, two major targeted enrichment strategies are available, i.e. PCR-based methods and hybridisation-based protocols. Although targeted PCR-based amplicon approach offers both easy workflow and shorter reaction time, requiring low DNA input at the same time, it suffers from several limitations, such as lower

\footnotetext{
${ }^{1}$ Department of Medical Genetics, Poznan University of Medical Sciences, Rokietnicka 8 Street, 60-806, Poznan, Poland. ${ }^{2}$ Postgraduate School of Molecular Medicine, Medical University of Warsaw, Żwirki i Wigury 61 Street, 02-091, Warsaw, Poland. ${ }^{3}$ Centers for Medical Genetics GENESIS, Grudzieniec 4 Street, 60-601, Poznan, Poland. ${ }^{4}$ Department of Biometry and Bioinformatics, Institute of Plant Genetics, Polish Academy of Sciences, Strzeszyńska 34, 60-479, Poznań, Poland. ${ }^{5}$ Perlan Technologies Sp. z o.o., Puławska 303 Street, 02-785, Warsaw, Poland. ${ }^{6}$ Department of Radiotherapy, The Maria Skłodowska Curie Memorial Cancer Centre and Institute of Oncology, Gliwice Branch, 44-101, Gliwice, Poland. *email: jamsheer@wp.pl
} 
sequencing complexity and coverage uniformity ${ }^{15-18}$. In general, the problem of non-specificity in PCR-based methods often cannot be circumvented by careful primer design, as the oligonucleotides have usually very short sequence ${ }^{16,18,19}$. On the other hand, an alternative approach, i.e. hybridisation-based enrichment protocols such as SureSelect (Agilent Technologies) is available and is commonly applied on the Illumina platforms. The SureSelect approach is based on biotinylated RNA oligomers of substantially greater length ( $120 \mathrm{bp})$, which can bind to DNA more specifically and consequently enrich the targeted regions of the genome, avoiding repetitive or non-specific amplification. Therefore, SureSelect enrichment strategy allows for obtaining better sequencing complexity and coverage uniformity ${ }^{16}$.

To our knowledge, SureSelect libraries have not been used so far to carry out the sequencing on the Ion Torrent S5 semiconductor-based platform. In this report, we present the first example of a successful adaptation of the hybridisation-based SureSelect enrichment protocol to the sequencing on the Ion Torrent S5 system. In addition, using a cohort of patients presenting with craniosynostosis, we emphasise the utility of targeted gene panel sequencing in the diagnostics of this aetiologically heterogeneous condition.

\section{Craniosynostosis}

Craniosynostosis (CS), premature fusion of one or more cranial sutures, occurs either as an isolated malformation or in a syndromic form, representing a genetically heterogeneous and clinically variable group of disorders ${ }^{20}$. Routine diagnostic screening of common craniosynostosis-associated genes (usually FGFR1, FGFR2, FGFR3, TWIST1 and often EFNB1, TCF12) enables to establish genetic aetiology in $21 \%^{21}$ to $62 \%{ }^{22}$, depending on the size of the study, ethnicity of the population, and range of the molecular analysis (either hot-spot screening or the entire gene sequencing). Since targeted NGS is regarded as a useful diagnostic method in identification of causative variants, especially in genetically heterogeneous disease ${ }^{23,24}$, we have designed and applied a custom hybridisation-based panel (Agilent Technologies) to screen CS patients with negative results of preliminary molecular screening (involving hot-spot mutations located in exon 7 of FGFR1, exons 7 and 8 of FGFR2, and exon 7 of FGFR3, as well as we analysed the entire coding sequence of TWIST1).

\section{Results}

Clinical description. We used an NGS targeted gene panel approach to screen 16 consecutive patients with CS in whom the result of conventional Sanger sequencing of preliminary molecular screening was negative. Distribution of prematurely fused sutures was as follows: coronal - 6/16 (unilateral - 4, bilateral - 2), metopic $5 / 16$, sagittal $-3 / 16$, multiple $-2 / 16.56 .25 \%$ of patients from our cohort presented with the syndromic form of CS, whereas $43.75 \%$ had an isolated defect. All patients were subjected to a careful dysmorphological assessment upon which clinically recognisable craniofacial malformations and other defects were photographically documented. Additionally, diagnostic imaging, including X-rays, CT scans, or head MRI was performed. DNA was extracted from venous blood samples of index patients and their parents.

Custom gene panel. On the basis of clinical reports available in medical literature and databases (OMIM, MGI) we have chosen the gene and variant content and designed a hybridisation-based panel comprised of 61 genes (see Table 1) and 11 SNVs (see Table 2) thought to be associated with craniosynostosis and abnormalities of craniofacial development. To create our gene panel we have used SureDesign software (Agilent Technologies, SantaClara, USA). The designed panel was further refined in collaboration with Perlan Biotechnologies. The panel summary is as follows: Agilent Design ID: 3056721, panel name: Cranio_V1, region size: $173.794 \mathrm{~kb}, 6033$ probes $\left(225709 \mathrm{~kb}\right.$ ) with region extension: 25 bases from $3^{\prime}$ end and 25 bases from $5^{\prime}$ end. The panel was classified to price tier 1, in which target region size ranges from 1 to $499 \mathrm{kbs}$. Hence, the target sequence and also the gene content could be increased at least two times without additional cost.

Quality control and coverage estimation. In each sample the estimated coverage exceeded 50 reads for over $95 \%$ of the target gene sequence (see Fig. 1). Mean coverages for the analysed genes and single nucleotide variants (SNVs) are summarised in Supplementary Materials (see Supplementary 1). There were marked discrepancies among the mean coverages of different samples, ranging from 129 in sample 3 to 337 in sample 11, with an average coverage of 240 reads calculated per gene. Across individual genes, SMAD6 had the lowest average coverage of 133 , while POLR1D was relatively best covered ( 321 on average).

Identification and evaluation of candidate variants. After sequencing of all 16 DNA samples on the Ion Torrent S5 system and completing the alignments, we assessed variant quality using multiple criteria (see Methods) and predicted the significance of individual variants. During the quality control, out of 2565 called variants, 87 (3.4\%) were dropped as artefacts. In three cases, we detected the variants definitely causative for the patients' phenotypes. Patient 1 was suspected of Pfeiffer syndrome, based on the clinical assessment. His phenotype involved sagittal CS, maxillary hypoplasia, high palate, proptosis, broad halluces, and skin syndactyly of $2^{\text {nd }}$ and $3^{\text {rd }}$ toes. X-ray examination of the feet showed hypoplastic middle phalanges of all toes and the relative widening of $1^{\text {st }}$ metatarsals as well as broadening of all bones forming the halluces (see Fig. 2a,b). Upon NGS analysis we found a pathogenic heterozygous variant in FGFR2 gene NM_000141.4:c.868T > G, NP_000132.3:p.Trp290Gly (HGMD: CM950464, ClinVar: 13284) (see Fig. 2c,d). Pathogenic variant was confirmed by means of Sanger sequencing in the index case and excluded in his unaffected parents, clearly indicating a de novo occurrence.

As female Patient 7 presented with complex CS involving sagittal and bilateral coronal synostosis, dolichocephaly, macrocephaly, prominent forehead, flat facial profile, proptosis, brachydactyly and broad halluces, clinical diagnosis also matched Pfeiffer syndrome (see Fig. 3a-c). At a molecular level, we identified a pathogenic heterozygous variant in FGFR2 NM_000141.4: c.1694A > G, NP_000132.3:p.Glu565Gly (HGMD: CM043278, 


\begin{tabular}{|c|c|c|c|c|c|}
\hline Gene & $\begin{array}{l}\text { HGNC } \\
\text { ID }\end{array}$ & $\begin{array}{l}\text { Reference } \\
\text { sequence number }\end{array}$ & Disorder (\#OMIM) & $\begin{array}{l}\text { Mode of } \\
\text { inheritance }\end{array}$ & Inclusion support \\
\hline$A L P L$ & 438 & NM_000478 & Different forms of hypophosphatasia & AR & Clinical evidence (OMIM, Pubmed) \\
\hline$A L X 1$ & 1494 & NM_006982 & Frontonasal dysplasia 3 (613456) & AR & Clinical evidence (OMIM, Pubmed) \\
\hline$A L X 3$ & 449 & NM_006492 & Frontonasal dysplasia 1 (136760) & AR & Clinical evidence (OMIM, Pubmed) \\
\hline \multirow{3}{*}{$A L X 4$} & \multirow{3}{*}{450} & \multirow{3}{*}{ NM_021926 } & Frontonasal dysplasia 2 (613451), & AR & \multirow{3}{*}{ Clinical evidence (OMIM, Pubmed } \\
\hline & & & Parietal foramina 2 (609597), & $\mathrm{AD}$ & \\
\hline & & & \{Craniosynostosis 5, susceptibility to $\}$ (615529) & $\mathrm{AD}$ & \\
\hline$B M P 4$ & 1071 & NM_001202 & Craniofacial development & & Literature review (Pubmed, MGI) \\
\hline CYP26B1 & 20581 & NM_019885 & $\begin{array}{l}\text { Craniosynostosis with radio humeral fusions and other } \\
\text { skeletal and craniofacial anomalies (614416) }\end{array}$ & AR & Clinical evidence (OMIM, Pubmed) \\
\hline DHODH & 2867 & NM_001361 & Miller syndrome (263750) & AR & Clinical evidence (OMIM, Pubmed) \\
\hline DPH1 & 3003 & NM_001383 & $\begin{array}{l}\text { Developmental delay with short stature, dysmorphic } \\
\text { features, and sparse hair (616901) }\end{array}$ & AR & Clinical evidence (OMIM, Pubmed) \\
\hline EDN3 & 3178 & NM_207034 & Craniofacial development & & Literature review (Pubmed) \\
\hline EDNRB & 3180 & NM_000115 & Craniofacial development & $\mathrm{AD}, \mathrm{AR}$ & Literature review (MGI, Pubmed) \\
\hline EFNA4 & 3224 & NM_005227 & Nonsyndromic coronal craniosynostosis & $\mathrm{AD}$ ? & Literature review (OMIM, Pubmed) \\
\hline EFNB1 & 3226 & NM_004429 & Craniofrontonasal syndrome (304110) & $\mathrm{XD}$ & Clinical evidence (OMIM, Pubmed) \\
\hline EFTUD2 & 30858 & NM_004247 & $\begin{array}{l}\text { Mandibulofacial dysostosis, Guion-Almeida type } \\
\text { (603892) }\end{array}$ & $\mathrm{AD}$ & Clinical evidence (OMIM, Pubmed) \\
\hline$E R F$ & 3444 & NM_006494 & Craniosynostosis 4 (600775) & $\mathrm{AD}$ & Clinical evidence (OMIM, Pubmed) \\
\hline ESCO2 & 27230 & NM_001017420 & Roberts syndrome (268300) & AR & Clinical evidence (OMIM, Pubmed) \\
\hline \multirow{5}{*}{ FGFR1 } & \multirow{5}{*}{3688} & \multirow{5}{*}{ NM_023110 } & Hartsfield syndrome (615465) & \multirow{5}{*}{$\mathrm{AD}$} & \multirow{5}{*}{ Clinical evidence (OMIM, Pubmed) } \\
\hline & & & Jackson-Weiss syndrome (123150) & & \\
\hline & & & Osteoglophonic dysplasia (166250) & & \\
\hline & & & Pfeiffer syndrome (101600) & & \\
\hline & & & Trigonocephaly 1 (190440) & & \\
\hline \multirow{11}{*}{ FGFR2 } & \multirow{11}{*}{3689} & \multirow{11}{*}{ NM_000141 } & $\begin{array}{l}\text { Antley-Bixler syndrome without genital anomalies or } \\
\text { disordered steroidogenesis (207410) }\end{array}$ & \multirow{11}{*}{$\mathrm{AD}$} & \multirow{11}{*}{ Clinical evidence (OMIM, Pubmed } \\
\hline & & & Apert syndrome (101200) & & \\
\hline & & & Beare-Stevenson cutis gyrata syndrome (123790) & & \\
\hline & & & Bent bone dysplasia syndrome (614592) & & \\
\hline & & & Craniofacial-skeletal-dermatologic dysplasia (101600) & & \\
\hline & & & Crouzon syndrome (123500) & & \\
\hline & & & Jackson-Weiss syndrome (123150) & & \\
\hline & & & LADD syndrome (149730) & & \\
\hline & & & Pfeiffer syndrome (101600) & & \\
\hline & & & Saethre-Chotzen syndrome (101400) & & \\
\hline & & & $\begin{array}{l}\text { Scaphocephaly, maxillary retrusion, and mental } \\
\text { retardation (609579) }\end{array}$ & & \\
\hline \multirow{7}{*}{ FGFR3 } & \multirow{7}{*}{3690} & \multirow{7}{*}{ NM_000142 } & $\begin{array}{l}\text { Achondroplasia (100800) } \\
\text { Crouzon syndrome with acanthosis nigricans (612247) }\end{array}$ & \multirow{7}{*}{$\mathrm{AD}$} & \multirow{7}{*}{ Clinical evidence (OMIM, Pubmed } \\
\hline & & & Hypochondroplasia (146000) & & \\
\hline & & & LADD syndrome (149730) & & \\
\hline & & & Muenke syndrome (602849) & & \\
\hline & & & SADDAN (616482) & & \\
\hline & & & Thanatophoric dysplasia, type I (187600) & & \\
\hline & & & Thanatophoric dysplasia, type II (187601) & & \\
\hline FIG4 & 16873 & NM_014845 & Yunis-Varon syndrome (216340) & AR & Clinical evidence (OMIM, Pubmed) \\
\hline FLNB & 3755 & NM_001457 & Larsen syndrome (150250) & $\mathrm{AD}$ & Clinical evidence (OMIM, Pubmed) \\
\hline FREM1 & 23399 & NM_144966 & Trigonocephaly 2 (614485) & $\mathrm{AD}$ & Clinical evidence (OMIM, Pubmed) \\
\hline GDF5 & 4220 & NM_000557 & Multiple synostoses syndrome (610017) & $\mathrm{AD}$ & Clinical evidence (OMIM, Pubmed) \\
\hline GLI3 & 4319 & NM_000168 & Greig cephalopolysyndactyly syndrome (175700) & $\mathrm{AD}$ & Clinical evidence (OMIM, Pubmed) \\
\hline IFT122 & 13556 & NM_052985 & Cranioectodermal dysplasia 1 (218330) & AR & Clinical evidence (OMIM, Pubmed) \\
\hline IFT140 & 29077 & NM_014714 & $\begin{array}{l}\text { Short-rib thoracic dysplasia } 9 \text { with or without } \\
\text { polydactyly (266920), }\end{array}$ & AR & Clinical evidence (OMIM, Pubmed) \\
\hline IFT43 & 29669 & NM_052873 & Cranioectodermal dysplasia 3 (614099) & AR & Clinical evidence (OMIM, Pubmed) \\
\hline IFT52 & 15901 & NM_001303458 & $\begin{array}{l}\text { Short-rib thoracic dysplasia } 16 \text { with or without } \\
\text { polydactyly (61702) }\end{array}$ & AR & Clinical evidence (OMIM, Pubmed) \\
\hline$I H H$ & 5956 & NM_002181 & $\begin{array}{l}\text { Copy number variations cause craniosynostosis } \\
\text { Philadelphia type (185900) }\end{array}$ & $\mathrm{AD}$ & Clinical evidence (OMIM, Pubmed) \\
\hline
\end{tabular}




\begin{tabular}{|c|c|c|c|c|c|}
\hline Gene & $\begin{array}{l}\text { HGNC } \\
\text { ID }\end{array}$ & \begin{tabular}{|l|} 
Reference \\
sequence number
\end{tabular} & Disorder (\#OMIM) & $\begin{array}{l}\text { Mode of } \\
\text { inheritance }\end{array}$ & Inclusion support \\
\hline IL11RA & 5967 & NM_001142784 & Craniosynostosis and dental anomalies (614188) & $\mathrm{AR}$ & Clinical evidence (OMIM, Pubmed) \\
\hline MASP1 & 6901 & NM_139125 & 3MC syndrome 1 (257920) & $\mathrm{AR}$ & Clinical evidence (OMIM, Pubmed) \\
\hline MEGF8 & 3233 & NM_001410 & Carpenter syndrome 2 (614976) & AR & Clinical evidence (OMIM, Pubmed) \\
\hline MITF & 7105 & NM_000248 & $\begin{array}{l}\text { Coloboma, osteopetrosis, microphthalmia, } \\
\text { macrocephaly, albinism, and deafness syndrome } \\
\text { (617306) }\end{array}$ & AR & Clinical evidence (OMIM, Pubmed) \\
\hline \multirow{2}{*}{ MSX2 } & \multirow{2}{*}{7392} & \multirow{2}{*}{ NM_002449 } & Craniosynostosis, Boston type (604757) & \multirow{2}{*}{$\mathrm{AD}$} & \multirow{2}{*}{ Clinical evidence (OMIM, Pubmed) } \\
\hline & & & Parietal foramina $1(168500)$ & & \\
\hline NOG & 7866 & NM_005450 & Multiple synostoses syndrome (186500) & $\mathrm{AD}$ & Clinical evidence (OMIM, Pubmed) \\
\hline$P 4 H B$ & 8548 & NM_000918 & Cole-Carpenter syndrome (112240) & $\mathrm{AD}$ & Clinical evidence (OMIM, Pubmed) \\
\hline PAX3 & 8617 & NM_181457 & Craniofacial-deafness-hand syndrome (122880) & $\mathrm{AD}$ & Clinical evidence (OMIM, Pubmed) \\
\hline POLR1C & 20194 & NM_203290 & Treacher-Collins syndrome 3 (248390) & AR & Clinical evidence (OMIM, Pubmed) \\
\hline POLR1D & 20422 & NM_015972 & Treacher-Collins syndrome 2 (613717) & $\mathrm{AR} / \mathrm{AD}$ & Clinical evidence (OMIM, Pubmed) \\
\hline$P O R$ & 9208 & NM_000941 & Antley-Bixler syndrome (201750) & AR & Clinical evidence (OMIM, Pubmed) \\
\hline$R A B 23$ & 14263 & NM_183227 & Carpenter syndrome 1 (201000) & AR & Clinical evidence (OMIM, Pubmed) \\
\hline \multirow{3}{*}{ RECQL4 } & \multirow{3}{*}{9948} & \multirow{3}{*}{ NM_004260 } & Baller-Gerold syndrome (218600), & \multirow{3}{*}{ AR } & \multirow{3}{*}{ Clinical evidence (OMIM, Pubmed) } \\
\hline & & & Rothmund-Thomson syndrome (268400), & & \\
\hline & & & RAPADILINO syndrome (266280) & & \\
\hline RSPRY1 & 29420 & NM_133368 & $\begin{array}{l}\text { Spondyloepimetaphyseal dysplasia, Faden-Alkuraya } \\
\text { type (616723) }\end{array}$ & AR & Clinical evidence (OMIM, Pubmed) \\
\hline RUNX2 & 10472 & NM_001024630 & Cleidocranial dysplasia (119600) & $\mathrm{AD}$ & Clinical evidence (OMIM, Pubmed) \\
\hline SF3B4 & 10771 & NM_005850 & Acrofacial dysostosis, Nager type (154400) & $\mathrm{AD}$ & Clinical evidence (OMIM, Pubmed) \\
\hline SIX2 & 10888 & NM_016932 & Frontonasal dysplasia, sagittal synostosis (n/a) & $\mathrm{AD}$ & Literature review (Pubmed) \\
\hline SKI & 10896 & NM_003036 & Shprintzen-Goldberg syndrome (182212) & $\mathrm{AD}$ & Clinical evidence (OMIM, Pubmed) \\
\hline SMAD6 & 6772 & NM_005585 & $\{$ Craniosynostosis 7, susceptibility to\}(617439) & $\mathrm{AD}$ & Literature review (OMIM, Pubmed) \\
\hline SMURF1 & 16807 & NM_001199847 & $\begin{array}{l}\text { Sporadic metopic craniosynostosis, craniofacial } \\
\text { development }\end{array}$ & & Literature review (Pubmed, MGI) \\
\hline SOX10 & 11190 & NM_006941 & Craniofacial development & & Literature review (Pubmed, MGI) \\
\hline SPRY1 & 11269 & NM_001258038 & Craniofacial development & & Literature review (Pubmed, MGI) \\
\hline SPRY4 & 15533 & NM_030964 & Craniofacial development & & Literature review (Pubmed, MGI) \\
\hline TCF12 & 11623 & NM_207036 & Craniosynostosis 3 (615314) & $\mathrm{AD}$ & Clinical evidence (OMIM, Pubmed) \\
\hline TCOF1 & 11654 & NM_001135243 & Treacher-Collins syndrome 1 (154500) & $\mathrm{AD}$ & Clinical evidence (OMIM, Pubmed) \\
\hline TGFBR1 & 11772 & NM_004612 & Loeys-Dietz syndrome 1 (609192) & $\mathrm{AD}$ & Clinical evidence (OMIM, Pubmed) \\
\hline TGFBR2 & 11773 & NM_003242 & Loeys-Dietz syndrome 2 (610168) & $\mathrm{AD}$ & Clinical evidence (OMIM, Pubmed) \\
\hline TTR & 12405 & NM_000371 & Maxillonasal dysplasia, Binder type & $?$ & Clinical evidence (OMIM, Pubmed) \\
\hline \multirow{3}{*}{ TWIST1 } & \multirow{3}{*}{12428} & \multirow{3}{*}{ NM_000474 } & Craniosynostosis 1(123100) & \multirow{3}{*}{$\mathrm{AD}$} & \multirow{3}{*}{ Clinical evidence (OMIM, Pubmed) } \\
\hline & & & Robinow-Sorauf syndrome (180750) & & \\
\hline & & & Saethre-Chotzen syndrome (181400) & & \\
\hline WDR19 & 18340 & NM_025132 & Cranioectodermal dysplasia 4 (614378) & AR & Clinical evidence (OMIM, Pubmed) \\
\hline \multirow[b]{2}{*}{ WDR35 } & \multirow[b]{2}{*}{29250} & \multirow[b]{2}{*}{ NM_001006657 } & Cranioectodermal dysplasia 2 (613610) & \multirow[b]{2}{*}{ AR } & \multirow[b]{2}{*}{ Clinical evidence (OMIM, Pubmed) } \\
\hline & & & $\begin{array}{l}\text { Short-rib thoracic dysplasia } 7 \text { with or without } \\
\text { polydactyly }(614091)\end{array}$ & & \\
\hline ZIC1 & 12872 & NM_003412 & Craniosynostosis 6 (616602) & $\mathrm{AD}$ & Clinical evidence (OMIM, Pubmed) \\
\hline
\end{tabular}

Table 1. Genes included in craniosynostosis-associated custom panel. AD - autosomal dominant, AR autosomal recessive, $\mathrm{XD}$ - X-linked disorder.

ClinVar: 374823) (see Fig. 3d,e). Pathogenic variant was confirmed by means of Sanger sequencing in the index case and excluded in his unaffected parents, clearly indicating a de novo occurrence.

In Patient 15, affected by complex CS the defect was composed of bilateral coronal synostosis (complete right-sided and partial left-sided) as well as partial left-sided lambdoid synostosis, marked craniofacial asymmetry, hearing impairment, scoliosis, bilateral split foot malformation with syndactyly of the remaining postaxial toes, extremely short and hypoplastic thumbs and $5^{\text {th }}$ fingers, short $5^{\text {th }}$ metacarpals and valgus deformity of the right $2^{\text {nd }}$ finger we detected two pathogenic variants in RECQL4 gene NM_004260.3:c.308C > T NP_004251.3, p.Pro103Leu (HGMD: CM033805, Clinvar: 239755) and c.3062G > A, p.Arg1021Gln (HGMD: CM033810, ClinVar: 135147) (see Fig. 4a-j). The two variants were confirmed by Sanger sequencing.

Intellectual development was normal in all three presented patients. 


\begin{tabular}{|l|l|l|l|}
\hline SNV & Gene & Genomic region & Description \\
\hline rs1009355 & BBS9 & Chr7:33218763 & common intron variant; NM_198428.2:c.442 + 1560T > A \\
\hline rs10254116 & BBS9 & Chr7:33237489 & common intron variant, NM_198428.2:c.442 + 20286T > C \\
\hline rs10262453 & BBS9 & Chr7:33256039 & common intron variant, NM_198428.2:c.442 + 38836A > C \\
\hline rs1420154 & BBS9 & Chr7:33290931 & common intron variant, NM_198428.2:c.443-5917G > A \\
\hline rs142092 & n/a & Chr20:7093432 & common genomic variant, NC_000020.10:g.7093432T >C \\
\hline rs179753 & LINC01428 & Chr20:7151968 & common intron variant, NR_110609.1:n.298 + 12022C > T \\
\hline rs1884302 & n/a & Chr20: 7106289 & common genomic variant, NC_000020.10:g.7106289T >C \\
\hline rs4140470 & LINC01428 & Chr20:14371737 & common intron variant, NR_110609.1:n.164 + 14997T >C \\
\hline rs6054814 & LINC01428 & Chr20:7198501 & common intron variant, NR_110609.1:n.164 + 23975C > A \\
\hline rs6107929 & n/a & Chr20:7121672 & common intron variant, NC_000020.10:g.7121672A > G \\
\hline rs6140226 & LINC01428 & Chr20: 7226483 & common intron variant, NR_110609.1:n.117-3960G > A \\
\hline
\end{tabular}

Table 2. Common SNVs associated with non-syndromic sagittal craniosynostosis included in craniosynostosis-associated genes panel (based on Justice et al. ${ }^{46}$ ).

\section{Discussion}

Craniosynostoses encompass a group of distinct, clinically variable phenotypes. Since the first dysmorphological description of the disease by Wheaton in late $19^{\text {th }}$ century, researchers have been extensively investigating the molecular background of CS ${ }^{25}$. The first causative gene for this condition was identified by Jabs et al. in 1993, who described a pathogenic variant within MSX2 in a family affected by autosomal dominant $\mathrm{CS}^{26}$. In the next four years, a few novel genes - FGFR1, FGFR2, FGFR3, TWIST1 - have been linked to premature fusion of the cranial sutures ${ }^{27-31}$. Recently, the development of high-throughput NGS-based strategies allowed for unravelling of the molecular basis of the condition at an unprecedented scale, as it happened for newly described variants, e.g. within ERF, SMAD6, and TCF12 $2^{32-34}$. However, in a significant percentage of cases, pathogenesis of the craniosynostosis is still unknown or only partially understood ${ }^{21,35,36}$. Thus, there is an unquestionable need of further research by means of WES or WGS to find novel genes or non-coding variants responsible for the development of CS in humans. In the diagnostic setting, however NGS-based panel approach appears to be a sufficient solution for mutational screening of all known causative genes or variants.

Here, we proved that a custom NGS panel designed by us represents a useful and effective tool in the molecular diagnostics of patients with CS. We investigated 16 unrelated patients and provided a diagnosis at a molecular level for 3 (19\%) of them, demonstrating the high coverage and high quality of the sequencing data at the same time. In Patient 1 and 7, the pathogenic variants were previously described in individuals affected either by Crouzon or Pfeiffer syndromes. Clinical evaluation of our patients was consistent with the diagnosis of Pfeiffer syndrome ${ }^{37-39}$. Interestingly, the variant detected in Patient 7, who presented with complex CS, macrocephaly, prominent forehead, flat face, proptosis, and broad halluces may not only give rise to Pfeiffer or Crouzon syndromes with normal intellectual development, but also to a more severe cloverleaf skull phenotype with an early demise $^{40}$. A broad phenotypic spectrum resulting from the same pathogenic variant suggests a possibility of other yet unidentified genetic or environmental modifiers, as indicated by Oldridge and colleagues ${ }^{41}$.

Patient 15 carried two pathogenic RECQL4 variants, both described as causative for Rothmund-Thomson syndrome (RTS) in osteosarcoma association study ${ }^{42}$. Both variants are very likely to occur in patient 15 in trans orientation, as they were identified in heterozygous state in two different probands ${ }^{42}$. Additionally, both p.Arg1021Pro and p.Pro103Leu RECQL4 alterations represent rare or extremely rare founder mutations already described in GnomAD/ExAC database. The variant p.Arg1021Pro was annotated in heterozygosity in 2 out of almost 280 thousand control alleles, while the variant p.Pro103Leu in 168 out of about 280 thousand alleles, clearly suggesting that those two SNVs do not represent a common haplotype. Importantly, none of the variants was found in homozygosity in a control healthy population, additionally indicating high likelihood of their pathogenicity. Unfortunately, we were unable to check the parental status for the RECQL4 mutations, as the parents disagree to undergo genetic testing. Interestingly, mutations within RECQL4 gene are linked to three distinct autosomal recessive conditions with overlapping phenotypes, i.e. Rothmund-Thomson, RAPADILINO, and Baller-Gerold syndrome (BGS), but only the last disorder comprises hearing loss in its clinical spectrum ${ }^{43-45}$. Considering lack of poikiloderma and the presence of hypoacusis in Patient 15, our final diagnosis was BGS. With clinical and molecular data presented here, we have broadened the phenotypic spectrum of previously reported RECQL4 alterations that may give rise to either RTS or BGS phenotype.

Although we confirmed the molecular diagnosis only in 3 out of 16 probands, the diagnostic yield of $19 \%$ is equal to some of the published research reports $(21 \%)^{21}$. Importantly, we analysed only the patients with negative results of Sanger sequencing for TWIST1 alterations and the most common pathogenic hot-spot variants of FGFR1, FGFR2, FGFR3. Consequently, our diagnostic score was significantly lower than e.g. $62 \%$ reported by Paumard-Hernández et al., who did not perform any molecular prescreening in the analysed individuals ${ }^{22}$.

Our study, in which a cohort of CS patients was utilised as an example, demonstrated the usefulness of targeted gene panel sequencing in the diagnostics of complex, genetically heterogeneous conditions. To our knowledge, we were the first to adapt SureSelect hybridisation-based enrichment protocol for the sequencing on the Ion Torrent S5 platform, which is intended to work preferably with amplicon-based panels (f.e. AmpliSeq ${ }^{\circledR}$ Thermo Fisher Scientific). Agilent hybridisation technology has not been previously used on Ion Torrent S5 equipment, hence the total cost of the analysis is higher than the standard ThermoFisher Scientific procedure. This is due to the fact that our experiment was 


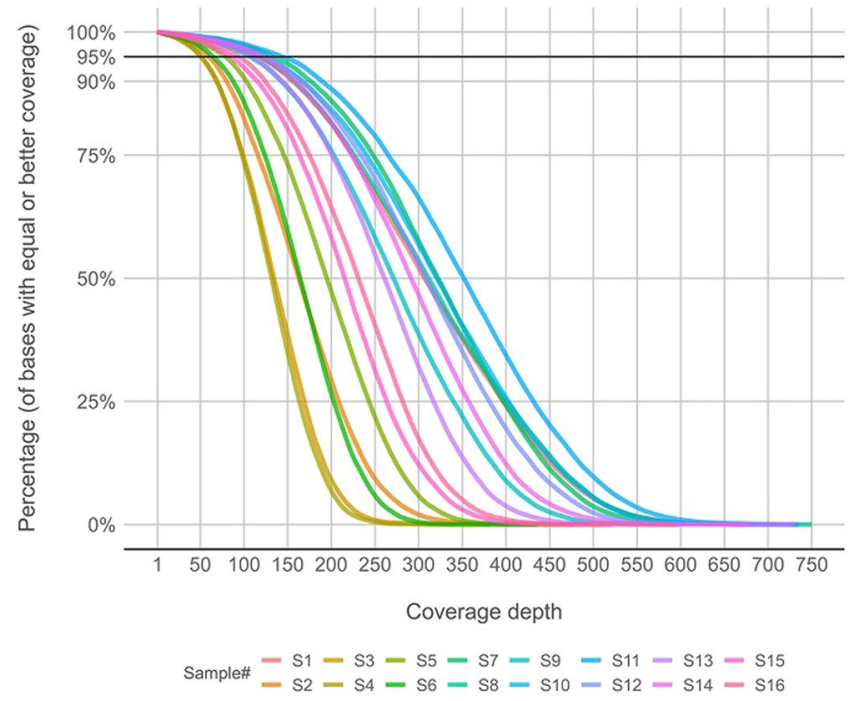

Figure 1. Comparison of per-base coverage depth for all samples. Additional horizontal line indicates $95 \%$ of total bases in panel target regions.
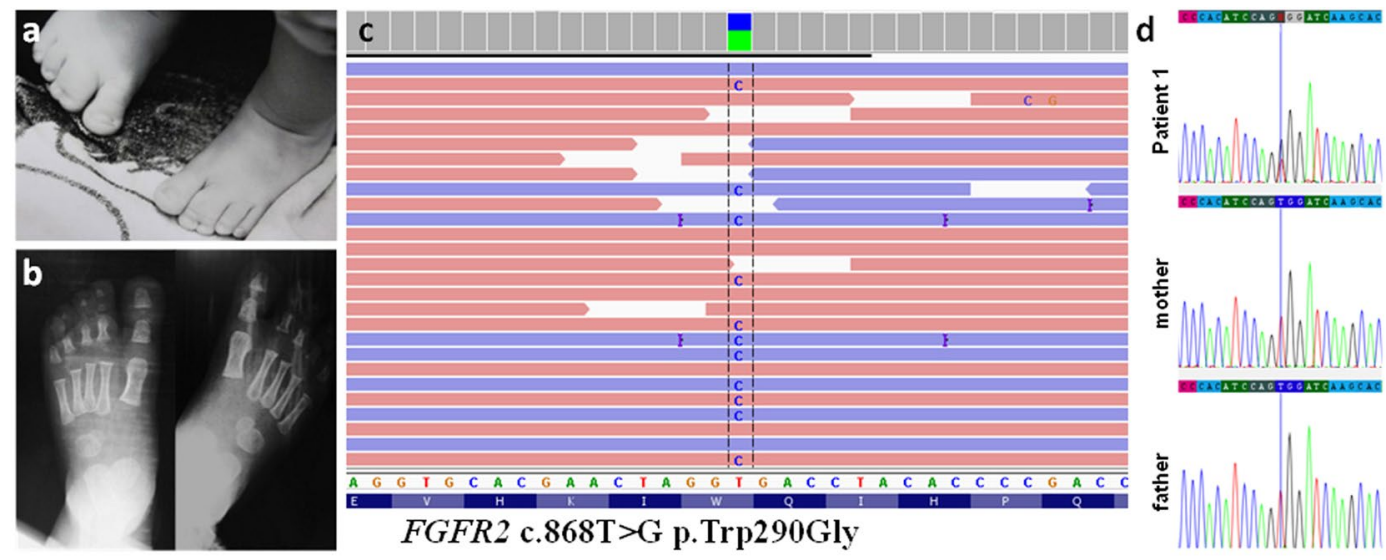

Figure 2. Clinical characteristics at the age of 12 months (a,b) as well as molecular results of Patient 1 (c,d). Patient 1, in addition to sagittal craniosynostosis, maxillary hypoplasia, high palate and proptosis, presented with broad halluces and skin syndactyly of $2^{\text {nd }}$ and $3^{\text {rd }}$ toes (a). X-ray of the feet showed small hypoplastic middle phalanges of all toes, relative widening of $1^{\text {st }}$ metatarsals and broadening of phalangeal bones forming halluces, and no bone syndactyly of $2^{\text {nd }}$ and $3^{\text {rd }}$ toes (b) Representation of the heterozygous FGFR2 deleterious variant c.868T > G p.Trp290Gly detected in Patient 1 by means of targeted next-generation sequencing (c) and validation studies of the proband and parental testing of the FGFR2 gene with the use of Sanger sequencing (d). Pathogenic variant c.868T $>$ G p.Trp290Gly was confirmed in the index case and excluded in his unaffected parents, clearly indicating a de novo occurrence.

focused on obtaining the most optimal quality of sequencing data and not on the cost reduction. The estimated price for the analysis was about 1.7 times higher per sample compared to the standard procedure, but optimisation of cost is certainly achievable. Although SureSelect hybridisation-based protocol is about 1.5 times more time consuming and represents a costlier alternative in comparison to amplicon-based approach, it provides several advantages, especially in the diagnostic setting, such as reduction of PCR-related edge artefacts, better and more exact matching of hybridisation probes. Consequently, it allows for obtaining higher specificity of the amplified region.

With the approach presented here, we achieved high molarity of both pooled libraries (756 and $525 \mathrm{pmol} / \mathrm{l})$ and exceeded coverage of 50 reads in each sample for over $95 \%$ of the target gene sequences $(173.794 \mathrm{~kb})$, as well as an average coverage of 240 reads per gene across all samples. Importantly, we obtained full coverage for all of the exons within FGFR2, which was not possible in amplicon-based protocols (e.g. Ampliseq ${ }^{\circledR}$, Thermo Fisher Scientific). Moreover, we were able to include all of the listed genes, which was impossible using even an advanced made-to order option in Ion AmpliSeq Designer tool (Thermo Fisher Scientific).

In conclusion, we successfully adapted hybridisation-based SureSelect enrichment protocol for the Ion Torrent S5 platform, demonstrating that an alternative enrichment strategy for library preparations can be applied prior 

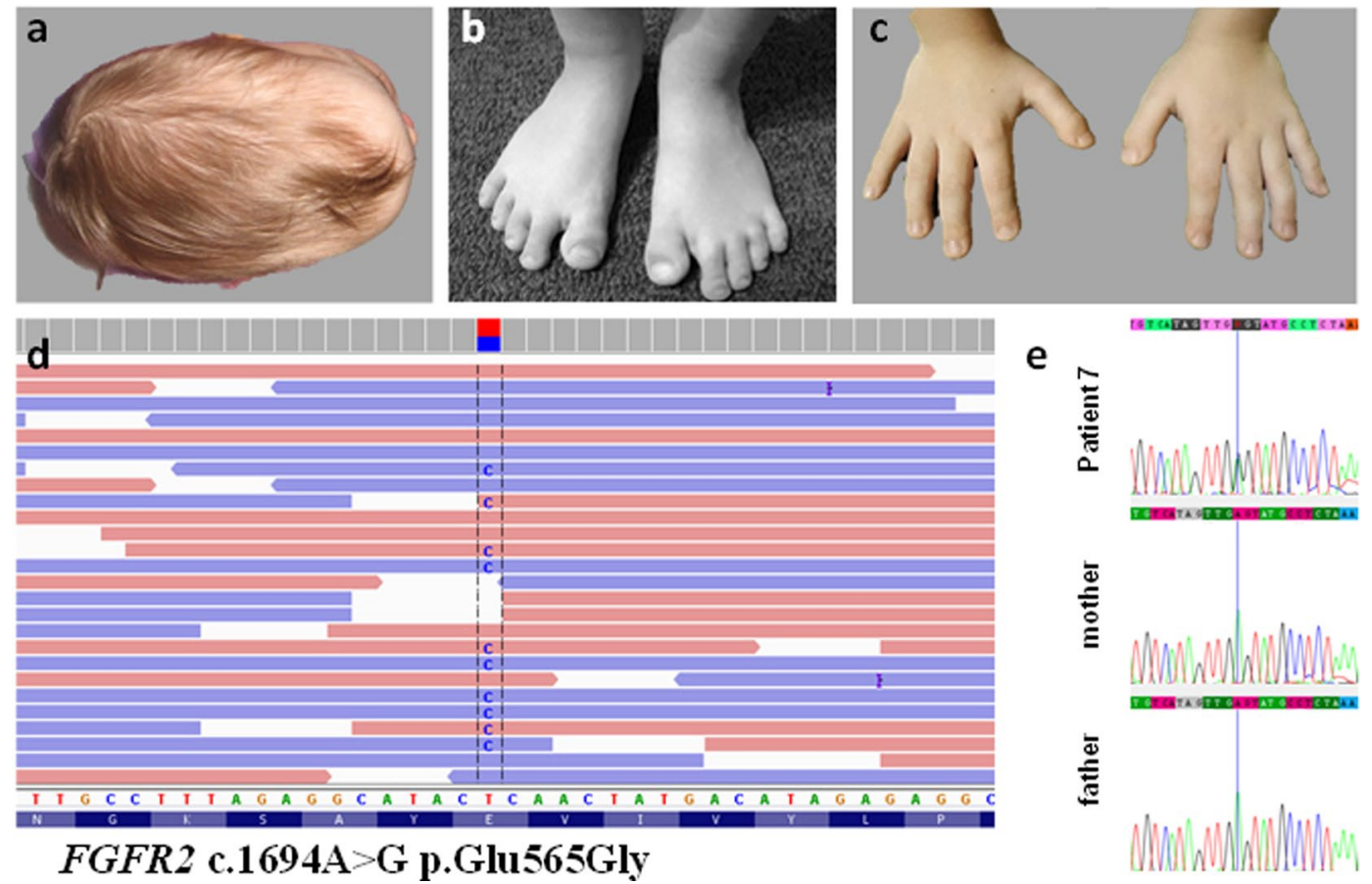

\section{$F G F R 2$ c.1694A $>$ G p.Glu565Gly}

Figure 3. Clinical characteristics at the age of 2 years (a) and 6 years $(\mathbf{b}, \mathbf{c})$ as well as molecular results of Patient 7 (d,e). Patient 7 presented with complex craniosynostosis involving sagittal and bilateral coronal synostosis, dolichocephaly, macrocephaly, prominent forehead (a), flat face, proptosis (full facial picture not shown), brachydactyly and broad halluces $(\mathbf{b}, \mathbf{c})$. Representation of the heterozygous FGFR2 deleterious variant c.1694A > G p.Glu565Gly unraveled in Patient 7 by means of targeted next-generation sequencing (d) and validation studies of the proband and parental testing of the FGFR2 gene with the use of Sanger sequencing (e). Pathogenic variant c.1694A > G p.Glu565Gly was confirmed in the index case and excluded in his unaffected parents, clearly indicating a de novo occurrence.

to sequencing on the Ion Torrent S5. Additionally, we proved the efficiency and clinical utility of the designed gene panel in the genetic testing of patients affected by variable CS.

\section{Methods}

All procedures involving human participants were performed in accordance with the ethical standards of the institutional and/or national research committee and with the 1964 Helsinki declaration and its later amendments or comparable ethical standards. Ethics approval was granted by the Institutional Review Board of Poznan University of Medical Sciences (no 742/17 obtained on $22^{\text {th }}$ June 2017). All patients and their parents agreed to participate in this study. This research involved human participants under the age of 18 years. Hence we obtained informed consents from parents and/or legal guardians. We present information or images that could lead to the identification of study participants. Accordingly, a specific consent has also been obtained from all parents and/or legal guardians for publication of identifying information/images in an online open-access publication.

Sample preparation. We extracted genomic DNA from the peripheral blood lymphocytes using the MagCore ${ }^{\circledR}$ HF16 Automated Nucleic Acid Extractor and quantified each gDNA using the Agilent Technologies TapeStation 4200 and Genomic DNA ScreenTape systems. The custom panel designed by us comprised 61 genes and 11 SNPs (see Tables 1 and 2) known to be involved in the development of craniofacial malformations, including craniosynostosis, in human and mouse. Prior to NGS, we performed targeted molecular screening of all patients for the common hot-spot mutations located in exon 7 of FGFR1 (c.755C > G p.Pro252Arg), exons 7 and 8 of FGFR2 (c.755C < G p.Ser252Trp and c.758C > G p.Pro253Arg), and exon 7 of FGFR3 (c.749C > G p.Pro250Arg) as well as we analysed the entire coding sequence of TWIST1 by means of Sanger sequencing. For NGS, we used high-molecular DNA with a range of DNA Integrity Number (DIN) 6.8 to 9.6. In the next step, Ion Shear Plus reagent (Thermo Fisher Scientific) cut each genomic DNA sample $(1 \mu \mathrm{g})$ into fragments of 50-250 bp. To obtain approximately $130 \mathrm{bp}$ peaks, we adjusted the time of incubation at $37^{\circ} \mathrm{C}$ to 50 minutes (step 1 ). Afterwards, we ligated each sample with Ion P1 Adapter and Ion Express barcode (Thermo Fisher Scientific). The ligation was as follows: 15 minutes at $25^{\circ} \mathrm{C}, 5$ minutes at $72^{\circ} \mathrm{C}$. We used a thermal cycler without a heated lid $\left(40^{\circ} \mathrm{C}\right)($ step 2$)$. Next, we proceeded to amplification of the adapter-ligated libraries through PCR reaction. To obtain an adequate yield for subsequent capture without introducing bias or non-specific products, we performed pre-capture PCR with Herculase II Fusion DNA Polymerase (Agilent Technologies) consisted of 8 cycles (step 3). After steps 1-3 we purified each sample with the use of Agencourt AMPure XP beads (Beckman Coulter Genomics), whereas after steps 1 and 3 we assessed the quality and quantity of samples on 4200 TapeStation using D1000 ScreenTape system (Agilent 

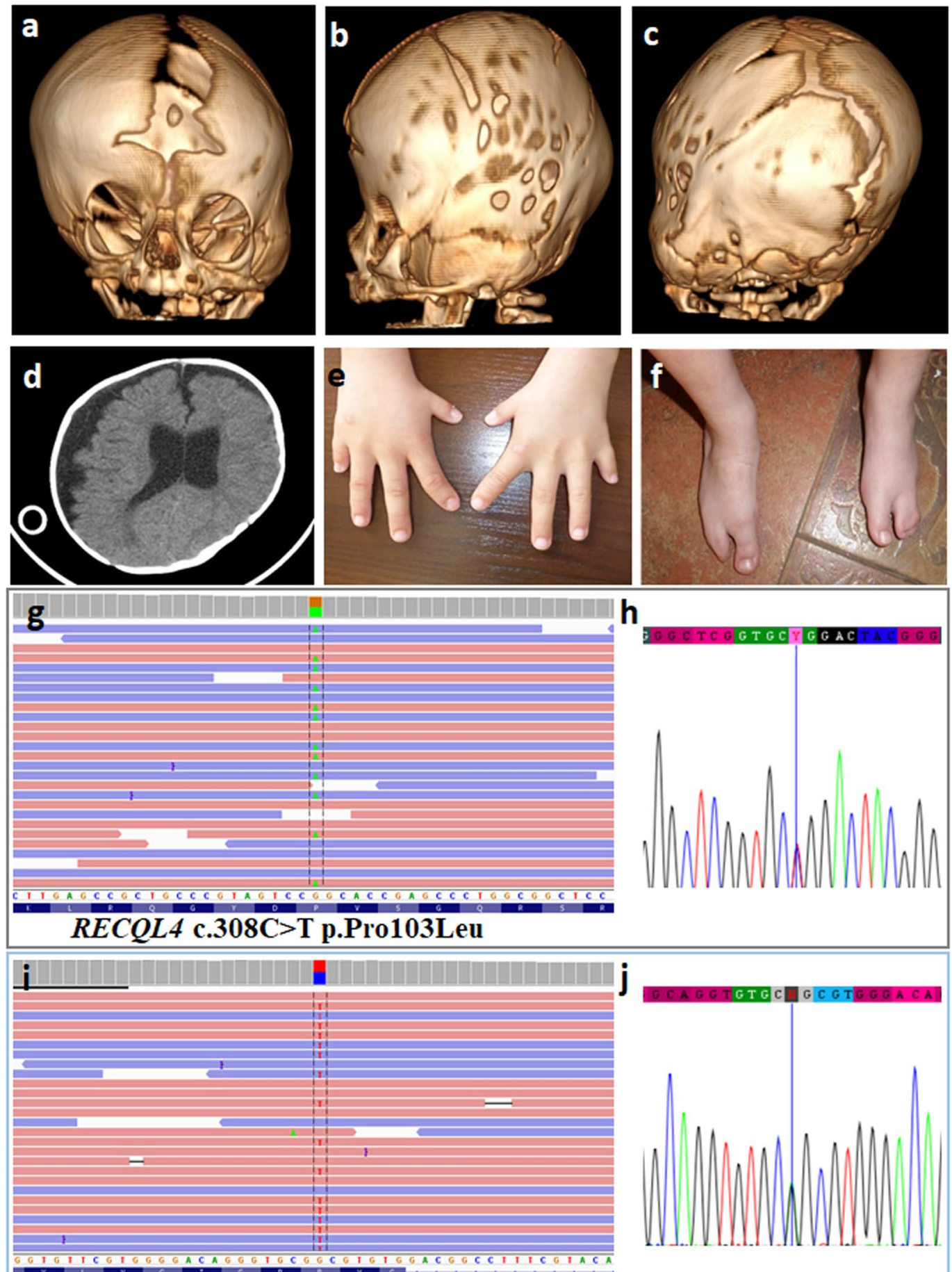

\section{RECQL4 c.3062G>A p.Arg1021Gln}

Figure 4. Clinical characteristics of Patient 15 at the age of $11(\mathbf{a}-\mathbf{d})$ and 9.5 years $(\mathbf{e}, \mathbf{f})$ as well as molecular results of the patient ( $\mathrm{g}-\mathrm{j})$. Patient 15 presented with complex craniosynostosis composed of bilateral coronal synostosis (complete right-sided and partial left-sided) as well as partial left-sided lambdoid synostosis shown in $3 \mathrm{D}$ modelling of the skull $(\mathbf{a}-\mathbf{c})$. CT scan of the head (d). Coronal sutures are prematurely fused. The right coronal suture is completely fused (a), while the left one is only partially fused (b); consequently, there is marked enlargement of the anterior fontanelle and widening of the sagittal suture, $(\mathbf{a}, \mathbf{c})$. Asymmetry of the skull and brain, including lateral ventricles, and enlargement of left subarachnoid space seen on horizontal section (d). Limb defect clinically recognized as bilateral split foot malformation with syndactyly of the remaining toes, extremely short and hypoplastic thumbs and $5^{\text {th }}$ fingers, short $5^{\text {th }}$ metacarpals and valgus deformity of the right $2^{\text {nd }}$ finger $(\mathbf{e}, \mathbf{f})$. Representation of the compound heterozygous RECQL4 deleterious variants c.308C $>\mathrm{T}$ p.Pro103Leu and 3062G > A p.Arg1021Gln detected in Patient 15 by means of targeted next-generation sequencing $(\mathbf{g}, \mathbf{i})$. Both pathogenic variants were confirmed with the use of Sanger sequencing $(\mathbf{h}, \mathbf{j})$. 
Technologies). In the first measurement of the samples (step 1) the obtained concentrations were between 0.229 and $6.39 \mathrm{ng} / \mu \mathrm{l}$, while in the final phase (step 3) concentrations ranged from 32.2 to $69.4 \mathrm{ng} / \mu \mathrm{l}$.

Hybridisation and capture. We prepared the NGS libraries for Ion Torrent S5 platform using hybridised capture-based target enrichment approach (SureSelect) developed by Agilent Technologies. We performed hybridisation of $750 \mathrm{ng}$ in $3.4 \mu \mathrm{l}$ of each genomic DNA library using SureSelect Target Enrichment Reagent Kit according to manufacturer's protocol for $<3 \mathrm{Mb}$ capture libraries. After 17 hours of hybridisation at $65^{\circ} \mathrm{C}$, we captured the targeted molecules on streptavidin-coated magnetic beads (Dynabeads MyOne Streptavidin T1).

Post-hybridisation amplification and sample processing for multiplexed sequencing. We amplified purified SureSelect-enriched DNA libraries and non-template control through PCR (11 cycles) with the use of Herculase II Fusion Polymerase. Before assessing DNA quality and quantity with High Sensitivity DNA Assay on TapeStation System, we purified each sample using AMPure XP beads. Based on the evaluated concentration of SureSelect-enriched DNA libraries, we calculated the amount of each sample to be included in the pool using the following formula: volume of barcoded sample: $\mathrm{V}(\mathrm{f}) \mathrm{xC}(\mathrm{f}) / \mathrm{nxC}(\mathrm{i})$. $\mathrm{V}(\mathrm{f})$ is the final required/ needed volume of the pool $(20 \mu \mathrm{l}), \mathrm{C}(\mathrm{f})$ is the initial concentration of all SureSelect-enriched DNA libraries in the pool, $\mathrm{n}$ is the number of samples to be combined, and C(i) is the initial concentration of each barcoded sample. To avoid the presence of additional fragments in each library, we size-selected our pools by agarose gel electrophoresis using the integrated E-Gel system (Thermo Fisher Scientific), purified them using Agencourt AMPure XP beads (Beckman Coulter Genomics) and finally validated using High sensitivity DNA assay on 4200 TapeStation. The molarity of pooled libraries was 756 and $525 \mathrm{pmol} / \mathrm{l}$, respectively (see Supplementary Fig. 1). Since the Ion Chef requires concentration of a loaded pooled library to be $50 \mathrm{pM}$, we diluted our samples using low TE buffer.

Emulsion PCR and sequencing. We subjected $25 \mu$ pooled libraries to emulsion PCR on the Ion Chef Instrument with the use of the Ion $520^{\mathrm{TM}} \& 530^{\mathrm{TM}} \mathrm{Kit}$, according to the manufacturer's protocol. Finally, we sequenced each loaded Ion $520^{\mathrm{TM}}$ chip on the Ion Torrent S5 System with the use of recommended reagents.

Sanger sequencing. We confirmed pathogenic variants using a conventional Sanger sequencing. We designed specific primers for the amplification using Primer3 tool (see Supplementary Table 1) and carried out the PCR reactions in a mixture containing the following substrates: DNA, $10 \times$ PCR Premix J buffer, primers, $\mathrm{H}_{2} \mathrm{O}$ and DNA polymerase. The PCR products were purified with Exonuclease I and shrimp alkaline phosphatase. Sequencing of the PCR product was carried out using dye-terminator chemistry (kit v.3, ABI 3130XL) and run on automated sequencer Applied Biosystems Prism 3700 DNA Analyser.

Bioinformatic analysis. Reads were initially demultiplexed and aligned to GrCh37 human reference sequence using the TorrentBrowser 5.0.4 software (Thermo Fisher Scientific) running as embedded instance within Ion Torrent S5 sequencer. The resulting alignment BAMs were further processed using IonReporter 5.2 pipeline (Thermo Fisher Scientific), which incorporated variant calling. Estimation of coverage for individual genes/positions was conducted via bedtools 2.27.1 (coverage subcommand) against a BED file defining coding parts of canonical transcripts (RefSeq mapped on UCSC hg19 reference; 5 bp padding around each exon included; see Supplementary 2). Variant quality control was assessed based on a fourfold metric (read depth - greater than or equal to 20, strandedness - no more than 4:1 difference in reporting of the variant on opposite strands, PHRED quality of over 30 , and variant proportion of not less than $15 \%$ of total reads). The existence of potentially significant variants was further reassessed through manual inspection of aligned reads in IGV 2.4 software.

Available clinical significance annotation was assessed in real-time from Human Gene Mutation Database Professional (https://portal.biobase-international.com/hgmd/pro/), ClinVar (https://www.ncbi.nlm.nih.gov/ clinvar/) and dbSNP (https://www.ncbi.nlm.nih.gov/snp) on 21/03/2018. The predictions for SIFT, PolyPhen and PhyloP (46-way) tools were retrieved from the IonReporter result files (tab-separated files). Frequency data was provided by Ensembl/VEP (software version 91, database version 91); additionally GNOMAD database was queried for homozygosity/heterozygosity of individual variants (http://gnomad.broadinstitute.org; version 2.0.2 of both exome and genome subsets; accessed on 21/03/2018 using tabix 1.5 software). The outcomes for Combined Annotation Dependent Depletion were obtained from CADD webserver (version 1.3, https://cadd.gs.washington.edu; accessed on 21/03/2018). MutationTaster results were obtained using 'query chromosomal position' options of the public webserver (http://www.mutationtaster.org; accessed on 21/03/2018). SnpEff prediction of variant consequences was obtained using local installation of SnpEff $4.3 \mathrm{t}$ with default databases for hg19 reference. The effect of substitutions on splicing was assessed using ADA + RF predictors available through dbNSFP v.3.5a (dbscSNV 1.1 dataset).

Visualisation of variants within gene/protein sequence context was done using R/Bioconductor package trackViewer (1.16.1, ran in R 3.4.1).

\section{Data availability}

BAMs files were submitted on Sequence Read Archive (SRA) NCBI (SRA accession: PRJNA597426 https://www. ncbi.nlm.nih.gov/sra/PRJNA597426; release date: 2020-07-01).

Received: 23 April 2019; Accepted: 18 February 2020;

Published online: 05 March 2020 


\section{References}

1. Zemojtel, T. et al. Phenotype Analysis of the Disease-Associated Genome. 6 (2015).

2. Bell, C. J. et al. Carrier Testing for Severe Childhood Recessive Diseases by Next-Generation Sequencing. Sci. Transl. Med. 3, 1-46 (2013).

3. Yang, Y. et al. Clinical Whole-Exome Sequencing for the Diagnosis of Mendelian Disorders. N. Engl. J. Med. 369, 1502-1511 (2013).

4. Glotov, A. S. et al. Targeted next-generation sequencing (NGS) of nine candidate genes with custom AmpliSeq in patients and a cardiomyopathy risk group. Clin. Chim. Acta 446, 132-140 (2015).

5. Thuresson, A.-C. et al. Whole genome sequencing of consanguineous families reveals novel pathogenic variants in intellectual disability. Clin. Genet. 1-4, https://doi.org/10.1111/cge.13470 (2018).

6. Endrullat, C., Glökler, J., Franke, P. \& Frohme, M. StandardiJzation and quality management in next-generation sequencing. Appl. Transl. Genomics 10, 2-9 (2016).

7. Buermans, H. P. J. \& den Dunnen, J. T. Next generation sequencing technology: Advances and applications. Biochim. Biophys. Acta - Mol. Basis Dis. 1842, 1932-1941 (2014).

8. Quail, M. A. et al. A tale of three next generation sequencing platforms: comparison of Ion Torrent, Pacific Biosciences and Illumina MiSeq sequencers. BMC Genomics 13, 1 (2012).

9. Aird, D. et al. Analyzing and minimizing PCR amplification bias in Illumina sequencing libraries. Genome Biol. 12, R18 (2011).

10. Feng, W. et al. Improving alignment accuracy on homopolymer regions for semiconductor-based sequencing technologies. BMC Genomics 17 (2016).

11. Bragg, L. M., Stone, G., Butler, M. K., Hugenholtz, P. \& Tyson, G. W. Shining a Light on Dark Sequencing: Characterising Errors in Ion Torrent PGM Data. PLoS Comput. Biol. 9 (2013).

12. Laehnemann, D., Borkhardt, A. \& Mchardy, A. C. Denoising DNA deep sequencing data - high-throughput sequencing errors and their correction. 1-26, https://doi.org/10.1093/bib/bbv029 (2015).

13. Warr, A. et al. Exome Sequencing: Current and Future Perspectives. G3 (Bethesda). 5, 1543-1550 (2015).

14. Meacham, F. et al. Identification and correction of systematic error in high-throughput sequence data. BMC Bioinformatics 12,451 (2011).

15. Ratan, A. et al. Comparison of sequencing platforms for single nucleotide variant calls in a human sample. PLoS One 8, e55089 (2013).

16. Samorodnitsky, E. et al. Evaluation of Hybridization Capture Versus Amplicon-Based Methods for Whole-Exome Sequencing. Hum. Mutat. 36, 903-914 (2015).

17. Chilamakuri, C. S. R. et al. Performance comparison of four exome capture systems for deep sequencing. BMC Genomics 15, 449 (2014).

18. Satya, R. V. \& DiCarlo, J. Edge effects in calling variants from targeted amplicon sequencing. BMC Genomics 15, 1073 (2014).

19. Merriman, B. \& Rothberg, J. M. Progress in Ion Torrent semiconductor chip. Electrophoresis 33, 3397-3417 (2012).

20. Wilkie, A. O. et al. Clinical dividends from the molecular genetic diagnosis of craniosynostosis. Am. J. Med. Genet. A. 143A, 1941-1949 (2007).

21. Wilkie, A. O. et al. Prevalence and complications of single-gene and chromosomal disorders in craniosynostosis. Pediatrics 126, e391-400 (2010).

22. Paumard-Hernández, B. et al. Expanding the mutation spectrum in 182 Spanish probands with craniosynostosis: Identification and characterization of novel TCF12 variants. Eur. J. Hum. Genet. 23, 907-914 (2015).

23. Mustafa, A. E. et al. Validation of Ion Torrent TM Inherited Disease Panel with the PGM TM Sequencing Platform for Rapid and Comprehensive Mutation Detection, https://doi.org/10.3390/genes9050267.

24. Mamanova, L. et al. Target-enrichment strategies for next-generation sequencing. Nat. Methods 7, 111-118 (2010).

25. SW, W. Two specimens of congenital cranial deformity in infants associated with fusion of the fingers and toes. Trans. Path. Soc. 45, 238-241 (1894).

26. Jabs, E. W. et al. A mutation in the homeodomain of the human MSX2 gene in a family affected with autosomal dominant craniosynostosis. Cell 75, 443-450 (1993).

27. el Ghouzzi, V. et al. Mutations of the TWIST gene in the Saethre-Chotzen syndrome. Nat. Genet. 15, 42-46 (1997).

28. Howard, T. D. et al. Mutations in TWIST, a basic helix-loop-helix transcription factor, in Saethre-Chotzen syndrome. Nat. Genet. 15, 36-41 (1997).

29. Muenke, M. et al. A common mutation in the fibroblast growth factor receptor 1 gene in Pfeiffer syndrome. Nat. Genet. 8, 269-274 (1994).

30. Reardon, W. et al. Mutations in the fibroblast growth factor receptor 2 gene cause Crouzon syndrome. Nat. Genet. 8, 98-103 (1994).

31. Meyers, G. A., Orlow, S. J., Munro, I. R., Przylepa, K. A. \& Jabs, E. W. Fibroblast growth factor receptor 3 (FGFR3) transmembrane mutation in Crouzon syndrome with acanthosis nigricans. Nat. Genet. 11, 462-464 (1995).

32. Sharma, V. P. et al. Mutations in TCF12, encoding a basic helix-loop-helix partner of TWIST1, are a frequent cause of coronal craniosynostosis. Nat. Genet. 45, 304-307 (2013).

33. Twigg, S. R. et al. Reduced dosage of ERF causes complex craniosynostosis in humans and mice and links ERK1/2 signaling to regulation of osteogenesis. Nat. Genet. 45, 308-313 (2013).

34. Timberlake, A. T. et al. Two locus inheritance of non-syndromic midline craniosynostosis via rare SMAD6 and common BMP2 alleles. Elife 5 (2016).

35. Katsianou, M. A., Adamopoulos, C., Vastardis, H. \& Basdra, E. K. Signaling mechanisms implicated in cranial sutures pathophysiology: Craniosynostosis. BBA Clin. 6, 165-176 (2016).

36. Timberlake, A. T. et al. De novo mutations in inhibitors of Wnt, BMP, and Ras/ERK signaling pathways in non-syndromic midline craniosynostosis. Proc. Natl. Acad. Sci. USA 114, E7341-E7347 (2017).

37. Park, W. J. et al. Novel FGFR2 mutations in Crouzon and Jackson-Weiss syndromes show allelic heterogeneity and phenotypic variability. Hum. Mol. Genet. 4, 1229-1233 (1995).

38. Machado, R. A. et al. RESEARCH LETTER A novel heterozygous mutation in FGFR2 gene causing Pfeiffer syndrome. 2838-2843, https://doi.org/10.1002/ajmg.a.38389 (2017).

39. C, G. P. D. \& Rajith, B. Predicting the Impact of Deleterious Mutations in the Protein Kinase Domain of FGFR2 in the Context of Function, Structure, and Pathogenesis - a Bioinformatics Approach. 1853-1870, https://doi.org/10.1007/s12010-013-0315-y (2013).

40. Kan, S. et al. Genomic Screening of Fibroblast Growth-Factor Receptor 2 Reveals a Wide Spectrum of Mutations in Patients with Syndromic Craniosynostosis. Am. J. Hum. Genet. 70, 472-486 (2002).

41. Oldridge, M. et al. Mutations in the third immunoglobulin domain of the fibroblast growth factor receptor-2 gene in Crouzon syndrome. Hum. Mol. Genet. 4, 1077-1082 (1995).

42. Wang, L. L. et al. Association between osteosarcoma and deleterious mutations in the RECQL4 gene Rothmund-Thomson syndrome. J. Natl. Cancer Inst. 95, 669-674 (2003).

43. Siitonen, H. A. et al. Molecular defect of RAPADILINO syndrome expands the phenotype spectrum of RECQL diseases. Hum. Mol. Genet. 12, 2837-2844 (2003).

44. Kitao, S. et al. Mutations in RECQL4 cause a subset of cases of Rothmund-Thomson syndrome. Nat. Genet. 22, 82-84 (1999). 
45. Van Maldergem, L. et al. Revisiting the craniosynostosis-radial ray hypoplasia association: Baller-Gerold syndrome caused by mutations in the RECQL4 gene. J. Med. Genet. 43, 148-152 (2006).

46. Justice, C. M. et al. A genome-wide association study identifies susceptibility loci for nonsyndromic sagittal craniosynostosis near BMP2 and within BBS9. Nat. Genet. 44, 1360-1364 (2012).

\section{Acknowledgements}

We are grateful to the patients and their family members for participating in this study. We would thank Andreas Polten for the technical support. This work was supported by the grant from the Polish National Science Centre, Poland UMO-2016/23/N/NZ5/02577 to EB-O and by the grant from the National Centre for Research and Development LIDER/008/431/L-4/12/NCBR/2013 to AJ.

\section{Competing interests}

Bartosz Wojciechowicz is a full-time employee of Perlan sp. z o.o., Perlan Technologies is an authorised distributor of Agilent Technologies in Poland.

\section{Additional information}

Supplementary information is available for this paper at https://doi.org/10.1038/s41598-020-61048-5.

Correspondence and requests for materials should be addressed to A.J.

Reprints and permissions information is available at www.nature.com/reprints.

Publisher's note Springer Nature remains neutral with regard to jurisdictional claims in published maps and institutional affiliations.

(c) (i) Open Access This article is licensed under a Creative Commons Attribution 4.0 International License, which permits use, sharing, adaptation, distribution and reproduction in any medium or format, as long as you give appropriate credit to the original author(s) and the source, provide a link to the Creative Commons license, and indicate if changes were made. The images or other third party material in this article are included in the article's Creative Commons license, unless indicated otherwise in a credit line to the material. If material is not included in the article's Creative Commons license and your intended use is not permitted by statutory regulation or exceeds the permitted use, you will need to obtain permission directly from the copyright holder. To view a copy of this license, visit http://creativecommons.org/licenses/by/4.0/.

(C) The Author(s) 2020 